\title{
Influence of Alendronate and Endplate Degeneration to Single Level Posterior Lumbar Spinal Interbody Fusion
}

\author{
Sang-Mok Kim, Wootack Rhee, Seongil Ha, Jae Hyeon Lim, II Tae Jang \\ Department of Neurosurgery, Seoul Nanoori Hospital, Seoul, Korea
}

\begin{abstract}
Objective: Using alendronate after spinal fusion is a controversial issue due to the inhibition of osteoclast mediated bone resorption. In addition, there are an increasing number of reports that the endplate degeneration influences the lumbar spinal fusion. The object of this retrospective controlled study was to evaluate how the endplate degeneration and the bisphosphonate medication influence the spinal fusion through radiographic evaluation.

Methods: In this study, 44 patients who underwent single-level posterior lumbar interbody fusion (PLIF) using cage were examined from April 2007 to March 2009. All patients had been diagnosed as osteoporosis and would be recommended for alendronate medication. Endplate degeneration is categorized by the Modic changes. The solid fusion is defined if there was bridging bone between the vertebral bodies, either within or external to the cage on the plain X-ray and if there is less than $5^{\circ}$ of angular difference in dynamic X-ray.

Results: In alendronate group, fusion was achieved in $66.7 \%$ compared to $73.9 \%$ in control group (no medication). Alendronate did not influence the fusion rate of PLIF. However, there was the statistical difference of fusion rate between the endplate degeneration group and the group without endplate degeneration. A total of $52.4 \%$ of fusion rate was seen in the endplate degeneration group compared to $91.3 \%$ in the group without endplate degeneration. The endplate degeneration suppresses the fusion process of PLIF.

Conclusion: Alendronate does not influence the fusion process in osteoporotic patients. The endplate degeneration decreases the fusion rate.
\end{abstract}

Key Words: Alendronate $\cdot$ Lumbar vertebrae $\cdot$ Osteoporosis $\cdot$ Spinal fusion $\cdot$ Endplate degeneration

\section{INTRODUCTION}

Alendronate medication has been the standard treatment for patients with osteoporosis ${ }^{2,24)}$. Alendronate binds to resorption surfaces of the bone and is locally released during acidification, and the rise in concentration stops resorption and membrane ruffling without destroying the osteoclasts ${ }^{23}$. Alendronate altered the density distributions, thereby decreasing the area with a high fracture risk, resulting in increased vertebral strength ${ }^{7}$. Despite these good effects on treating osteoporosis, using alend-

- Received: April 23, 2014 • Revised: November 21, 2014

- Accepted: November 24, 2014

Corresponding Author: Sang-Mok Kim, MD, PhD

Department of Neurosurgery, Seoul Nanoori Hospital, 63-8, Nonhyun-

dong, Gangnam-gu, Seoul 135-010, Korea

Tel: +82-2-3446-9797, Fax: +82-2-3448-0213

E-mail: kimkeith@naver.com

®This is an Open Access article distributed under the terms of the Creative Commons Attribution Non-Commercial License (http://creativecommons.org/ licenses/by-nc/3.0/) which permits unrestricted non-commercial use, distribution, and reproduction in any medium, provided the original work is properly cited. ronate after spinal fusion is the controversial issue due to the inhibition of osteoclast mediated bone resorption. Many in vitro studies show the negative effect of alendronate to the lumbar spinal interbody fusion ${ }^{6,9,13)}$. However, recent prospective randomized control studies showed that alendronate overcame its detrimental biological effect on the healing process of spinal fusion 18. The endplate degeneration of the lumbar spine has been thought as the source of back pain, but it also influenced the lumbar spinal fusion ${ }^{12)}$. Signal intensity changes of vertebral endplates and subchondral bone are often observed in magnetic resonance imaging (MRI) in patients with spinal degenerative diseases. In 1988, Modic et al. ${ }^{16,17)}$ summarized these changes and classified them into three types, and then Modic changes (MC), as a medical term, were used in the studies on spinal degenerative diseases. Although the etiology of MC remains poorly understood, some progress has been achieved in basic research during the past two decades, including studies of the prevalence and some clinical significance.

What if there is the endplate degeneration at the fusion level, would activity of osteoclast increase or decrease to influence the fusion process? If the activity of osteoclast increase 
than is it reasonable to give alendronate to patient by expecting the inhibiting effect of osteoclast? If the activity of osteoclast decrease than is it harmful for giving alendronate to the patient?

The object of this retrospective study was to evaluate how the endplate degeneration and the alendronate medication influence the fusion rate of osteoporotic patient through radiographic evaluation.

\section{MATERIALS AND METHODS}

\section{Patient Population}

In this study, 44 patients who underwent single-level lumbar interbody fusion using cage were examined from April 2007 to March 2009. Nine patients were male and thirty-five patients were female. The patients' mean age was 64.7 years (range 60-74 years). The patients were diagnosed as degenerative spondylolisthesis, isthmic spondylolisthesis, severe intracanal stenosis, foraminal stenosis, and recurred herniated intervertebral disc (HIVD). The minimum X-ray follow up period was 2 years (range 2-5 years) and the mean follow up period was 33.8 months.

All patients were diagnosed as osteoporosis using computed tomography (CT) bone densitometry and recommended for pharmacological treatment. The diagnosis of osteoporosis was made according to the World Health Organization. Patients were divided into the alendronate group (alendronate sodium [Fosamax] $35 \mathrm{mg} /$ week) or the control group. The control group was classified as patients who were not taking alendronate for less than 1 week due to poor economic conditions and gastrointestinal trouble. Compared to the previous study ${ }^{18)}$, we did not give vitamin D, calcium supplement, and any other medication for osteoporosis to the control group. The mean preoperative lumbar spine BMC and T-score were $63.76 \mathrm{mg} / \mathrm{cc}$ and -3.75 in the alendronate group, and $69.58 \mathrm{mg} / \mathrm{cc}$ and -3.98 in the control group. There was no statistical difference between age and $\mathrm{BMC}$, and between the control and alendronate group.

\section{Radiographic Assessment}

Endplate degeneration is categorized by the Modic classification ${ }^{16,17)}$. Modic change type 1 [hypo-intense signal in T1-weighted imaging (T1WI) and hyperintense signal in T2weighted imaging (T2WI)] is corresponding to the vertebral body edema and hyper-vascularity. MC type 2 (hyperintense signal in T1WI and hyperintense signal in T2WI) is reflecting fatty replacements of the red bone marrow. MC type 3 (hypointense signal in T1WI and hypointense signal in T2WI) was consisted of subchondral bone sclerosis.
The plain static radiographs were examined by two different neurosurgeons. They were interpreted as demonstrating the presence of fusion if there was a bridging bone between the vertebral bodies either within or external to the cage ${ }^{22)}$. Fig. $1 \mathrm{~A}$ shows a typical radiograph assessed as demonstrating a solid fusion. Fig. 1B shows the features of a pseudarthrosis with discontinuity in the bone graft and lucency adjacent to the cage.

The flexion radiographs were taken with the patient in the standing position trying to bend forward as far as possible, whereas the extension radiographs were taken with the patient maximally arching his or her back. The spinal surgeon used the method advocated by Simmons ${ }^{15)}$, which involved identifying two landmarks on the anterior portion of the superior and inferior vertebral bodies, drawing a line through these landmarks, and measuring the angle subtended by the two resulting lines. An increase of $2^{\circ}$ in the acute angle made by these two lines on extension views was considered to be a sign of nonunion. The apparent fusion rate was also determined using this same method, except that the $5^{\circ}$ cutoff prescribed by the United States Food and Drug Administration (FDA) was used.

\section{RESULTS}

The chi-square test was used to determine whether there is a significant difference between two groups in Fig. 2 to 6 . The $\mathrm{p}$ value $(<0.01)$ was used to test statistical significance of evidence. The SPSS21(Statistical Package for the Social Sciences 21.0) was used to analyzed. Overall, $75 \%$ of fusion rate was seen and $25 \%$ of non-fusion rate was seen. In alendronate group, fusion was achieved in $66.7 \%$ compared to $73.9 \%$ in control group (no medication) (Fig. 2). There was no statistical difference between the two groups $(p=0.599)$. Alendronate seems to have no effect on the fusion rate.

The endplate degeneration was classified by Modic ${ }^{16,17)}$. A total of $52.4 \%$ of fusion rate was seen in the endplate degeneration group compared to $91.3 \%$ in the group without endplate
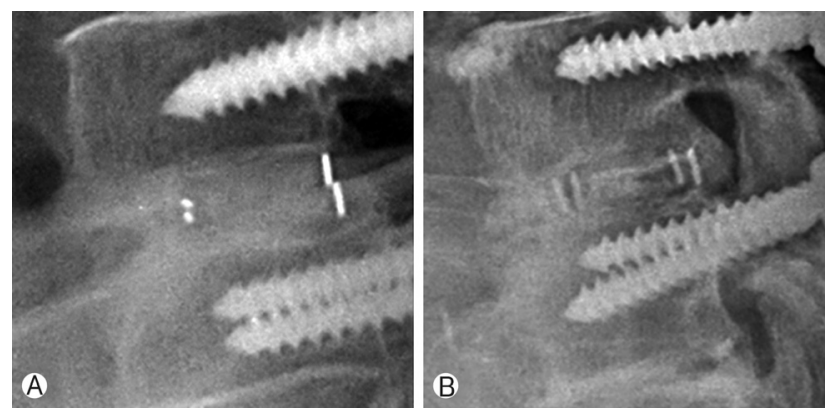

Fig. 1. (A) Plain static radiograph interpreted as a successful fusion. (B) Plain static radiograph interpreted as a non-fusion. 
degeneration (Fig. 3). There was a statistical difference of fusion rate between the endplate degeneration group and the group without endplate degeneration $(\mathrm{p}=0.599)$. Endplate degeneration decreases the fusion rate in PLIF. However, subtypes of $\mathrm{MC}$ do not influence the fusion rate. There was no statistical difference of fusion rate between the MC types ( $p=0.293$ ) (Fig. 4).

Comparison of alendronate effect on patient without endplate degeneration shows in Fig. 5. In alendronate group fusion was achieved in $92.3 \%$ of patients. In control group fusion was achieved in $90 \%$ of patients. There was no statistical difference between two groups $(\mathrm{p}=0.846)$. Alendronate did not influence the fusion rate of patients without endplate degeneration. Among patients with endplate degeneration fusion was achieved in $37.5 \%$ of patients in alendronate group. In control group fusion was achieved in $61.5 \%$ of patients (Fig.

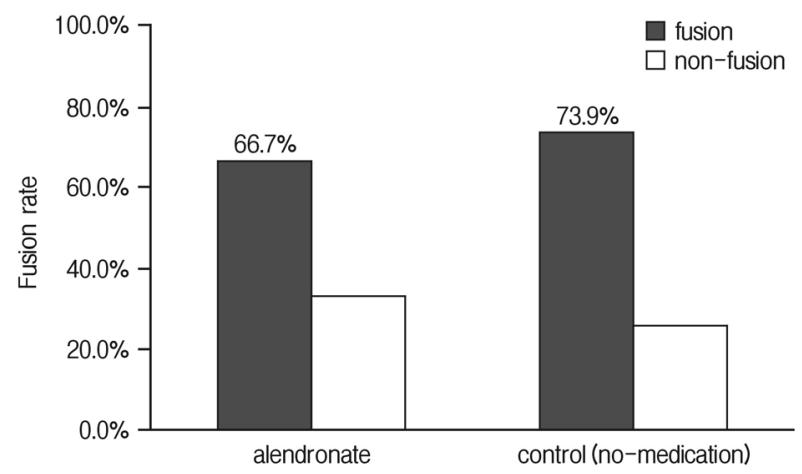

Fig. 2. Fusion rate of the group with alendronate medication and the group with no-medication. In alendronate group, fusion was achieved in $66.7 \%$ compared to $73.9 \%$ in control group (no medication). There was no statistical difference between the two groups $(p=0.599)$.

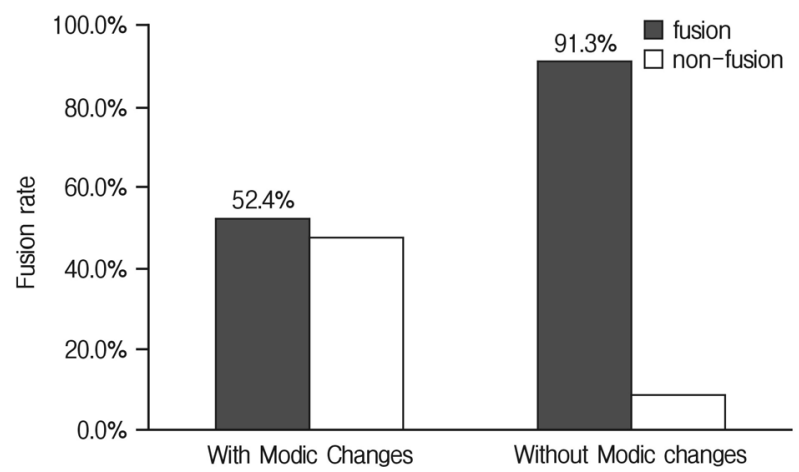

Fig. 3. Fusion rate in the group with Modic changes and without Modic changes. $52.4 \%$ of fusion rate was seen in the Modic changes group compared to $91.3 \%$ in the group without Modic changes. There was a statistical difference between the two groups $(p=0.004)$.
6). There was no statistical difference between two groups ( $p$ $=0.284$ ). Alendronate seems to have no effect on the fusion rate of the group with endplate degeneration.

\section{DISCUSSION}

The skeleton is a dynamic tissue that could remodel and repair the constant mirocracks that develop both in the cancellous bone and in the cortical bone for a lifetime ${ }^{8)}$. It had been assumed that the mechanisms of bone remodeling were likely to be different in cancellous versus cortical bone ${ }^{8}$. Specifically, the assumption was that the cells needed for bone remodeling traveled directly from the red marrow to the bone surfaces in cancellous bone, whereas they accessed cortical

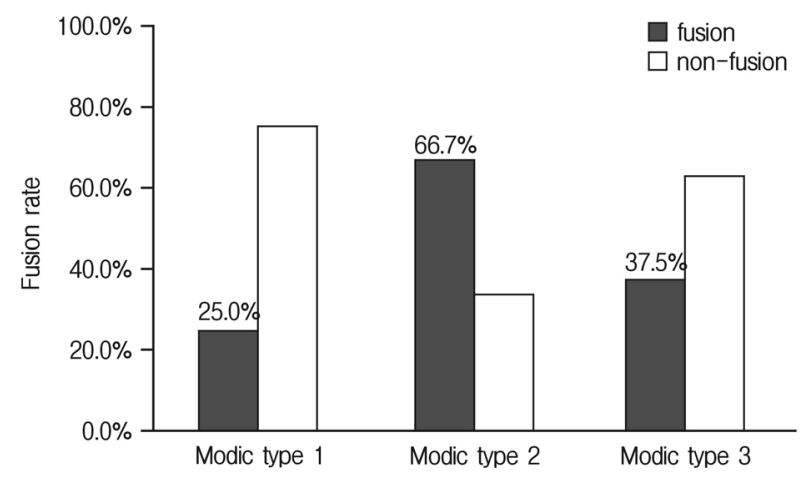

Fig. 4. Influence of Modic changes type to fusion rate. There was no statistical difference of fusion rate between Modic change types $(p=0.293)$.

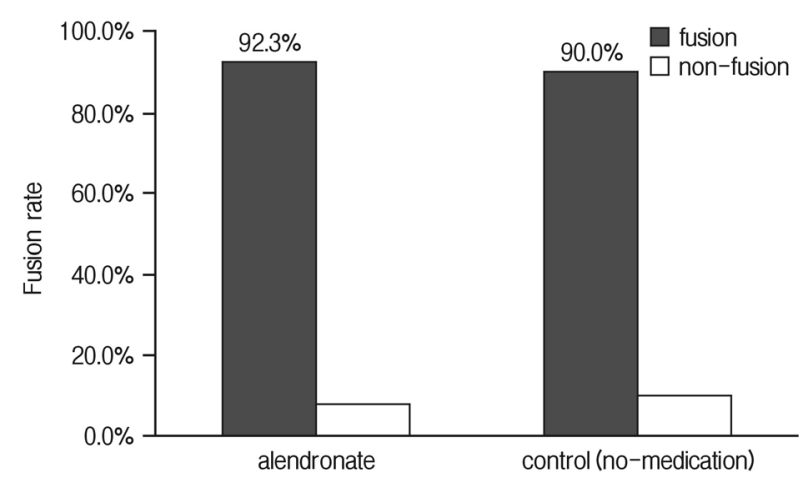

Fig. 5. Fusion rate of the group without Endplate degeneration (without Modic change). In alendronate group fusion was achieved in $92.3 \%$ of patients. In control group fusion was achieved in 90 $\%$ of patients. There was no statistical difference between two groups $(p=0.846)$. 


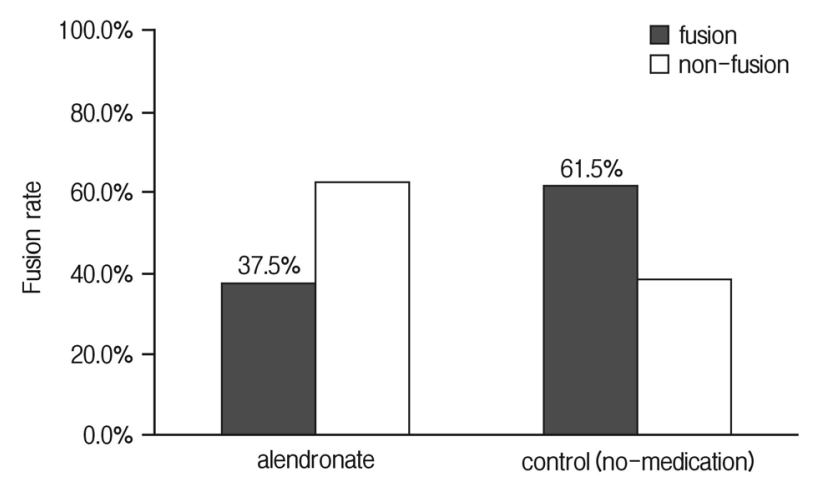

Fig. 6. Fusion rate of the group with Endplate degeneration (with Modic change). In alendronate group fusion was achieved in $37.5 \%$ of patients. In control group fusion was achieved in $61.5 \%$ of patients. There was no statistical difference between two groups $(p=0.284)$.

bone via the vasculature ${ }^{20)}$.

The successful spinal surgery is depending on gaining the long-stand spinal stability. Because of the spinal instrumentation gives short-term support, the solid bony fusion is necessary to obtain permanent stability. Coordination between bone formation and resorption is very important for spinal fusion.

The evaluation of factors that influence the lumbar spinal interbody fusion is very difficult to assess due to the complexity of biomechanics of each patient and difference in bone quality. Effects of spinal biomechanics are hard to quantify, whereas the bone quality can be easily measured by using bone densitometry and differentiated by using MRI according to Modic $^{16,17)}$. Most of spine surgeons believe that osteoporosisrelated bone fragility is the primary reason for implant fixation failure $^{11)}$, and it leads to fusion Failure ${ }^{4,14)}$.

Both osteoporosis and MC related spinal fusion rate has been known to be low compared to normal or osteopenic patients $^{12,18)}$. The only available human study shows osteoporosis and alendronate influence the spinal fusion rate ${ }^{18)}$. This study demonstrates a solid fusion was achieved in 95\% of patients in the alendronate group, and $65 \%$ of those in the control group $^{18)}$. Most animal studies ${ }^{1,3,5,13,19,21,26-29)}$ evaluating the impact of bisphosphonates on fusion rate have not found statistically significant changes with treatment, although this fact may be attributable to low statistical power. The animal literature does suggest that bisphosphonate therapy results in a less histologically mature fusion mass; however, the impact of these changes on fusion mass biomechanics is unclear. There are many studies about the endplate degeneration correlating with the symptoms, but few studies report a correlation between $\mathrm{MC}$ and spinal fusion. Kwon ${ }^{12)}$ shows that the bony fusion rate was $97 \%$ in patients without degeneration and $83 \%$ in patients with MC. The bony fusion rate in each group was $81 \%$ in type $1,84 \%$ in type 2 , and $55 \%$ in type 3 . The clinical success and fusion rates were significantly lower in patients with type 3 degeneration ${ }^{12)}$.

Generally accepted fusion rate of lumbar spine is around $85-95 \%{ }^{10)}$. In our study, the overall fusion rate was $75 \%$. Since half of the patients had MC in lumbar spine endplate, which could lower the fusion rate, fusion rate of our study could be acceptable.

We reviewed the osteoporotic patients into two groups. The fusion rate of the alendronate group was $76.2 \%$ compared to $73.9 \%$ of the control group. There was no statistical difference between the alendronate group and the control group. This result refutes the previous prospective randomized controlled study ${ }^{18)}$. From this result we could speculate that alendronate does not alter fusion rate in osteoporotic patients.

We reviewed the patients into endplate degeneration and without the degeneration groups. A total of $42.9 \%$ of non-fusion rate was seen in the endplate degeneration group, compared to $8.7 \%$ in the group without endplate degeneration. There was the statistical difference of fusion rate between the endplate degeneration group and the group without endplate degeneration. Endplate degeneration had potential to decrease the lumbar interbody fusion. We also compared the fusion rate of endplate degeneration by MC. There was no statistical difference of fusion rate between MC. The degeneration itself influenced the fusion rate, but there was no difference between fusion rates of the MC subtypes. Small sample size and surgical technique might influence the outcome. Most of the spinal surgeons use endplate decortications technique, a process of making microfracture of fusion bed, during lumbar interbody fusion. This process would eliminate the cortical bone and sclerotic endplate degeneration, such as MC type 3, to making bone marrow surface in fusion bed. However, endplate degeneration seems to influence the biological environment for those osteoclasts and osteoblasts. The limitation of this result is that the thickness of sclerotic endplate had not been measured. The thicker at the endplate sclerosis, the harder to make good fusion bed.

We also put both factors, alendronate and endplate degeneration, together to see how these factors influence the fusion rate of the lumbar spine. The effects of alendronate on osteoporotic patient with and without MC show that alendronate did not influence the fusion rate of patients whether patients had a MC or not.

The limitation of this study was that despite the wide use of flexion-extension radiography, the definition of a solid fusion still remains controversial. In the radiologic assessment of interbody fusion using carbon fiber cages, the use of plain radiographs and flexion-extension radiographs produced much higher fusion rates than assessment with thin-section helical 
computed tomography scans ${ }^{25)}$. The thin-section helical computed tomography studies demonstrated the radiographic presence or absence of bridging bone, a property that was not seen with plain static radiographs or flexion-extension radiographs $^{22)}$. Another limitation is the biomechanical loads on fusion level that could not be measured in number due to different lifestyle of each patient, such as jobs.

Finally, we still have several questions to be answered in future research: (1) How long should patients take bisphosphonates before and after spinal fusion surgery to improve the strength of vertebral bodies? (2) Could the current findings in a single-level posterior lumbar interbody fusion be applied to other situations, including multi-level spinal fusion, or correction of spinal deformities? (3) How will different types of spinal fusion, especially posterolateral lumbar fusion, affect the fusion process under alendronate medication? (4) Does bridging bone under alendronate treatment have sufficient mechanical properties and bone quality?

\section{CONCLUSION}

This study showed that alendronate does not influence the fusion process in osteoporotic patients. The endplate degeneration decreases the fusion rate, even though there was no difference in the MC subtypes. With the presence of endplate degeneration, alendronate does not influence the fusion rate in osteoporotic patients.

The effect of alendronate medication after the single level PLIF still remained unknown. The decortication technique is more important than medication in endplate degeneration at the fusion site.

\section{REFERENCES}

1. Babat LB, McLain R, Milks R, Ferrara L, Sohn MJ: The effects of the antiresorptive agents calcitonin and pamidronate on spine fusion in a rabbit model. The Spine Journal: Official Journal of the North American Spine Society 5:542-547, 2005

2. Diab DL, Watts NB: Bisphosphonates in the treatment of osteoporosis. Endocrinology and Metabolism Clinics of North America 41:487-506, 2012

3. Gezici AR, Ergun R, Gurel K, Yilmaz F, Okay O, Bozdogan $\mathrm{O}$ : The effect of risedronate on posterior lateral spinal fusion in a rat model. Journal of Korean Neurosurgical Society 46: 45-51, 2009

4. Hou Y, Yuan W: Influences of disc degeneration and bone mineral density on the structural properties of lumbar end plates. The Spine Journal: Official Journal of the North American Spine Society 12:249-256, 2012

5. Huang RC, Khan SN, Sandhu HS, Metzl JA, Cammisa FP, Jr.,
Zheng F, et al: Alendronate inhibits spine fusion in a rat model. Spine 30:2516-2522, 2005

6. Idris AI, Rojas J, Greig IR, Van't Hof RJ, Ralston SH: Aminobisphosphonates cause osteoblast apoptosis and inhibit bone nodule formation in vitro. Calcified Tissue International 82:191-201, 2008

7. Imai K, Ohnishi I, Matsumoto T, Yamamoto S, Nakamura K: Assessment of vertebral fracture risk and therapeutic effects of alendronate in postmenopausal women using a quantitative computed tomography-based nonlinear finite element method. Osteoporosis international: a journal established as result of cooperation between the European Foundation for Osteoporosis and the National Osteoporosis Foundation of the USA 20: 801-810, 2009

8. Khosla S, Westendorf JJ, Oursler MJ: Building bone to reverse osteoporosis and repair fractures. The Journal of Clinical Investigation 118:421-428, 2008

9. Koo KH, Lee JH, Chang BS, Lee CK: Effects of Alendronate on Lumbar Posterolateral Fusion Using Hydroxyapatite in Rabbits. Artificial Organs, 2012

10. Kornblum MB, Fischgrund JS, Herkowitz HN, Abraham DA, Berkower DL, Ditkoff JS: Degenerative lumbar spondylolisthesis with spinal stenosis: a prospective long-term study comparing fusion and pseudarthrosis. Spine 29:726-733; discussion 733-724, 2004

11. Kumano K, Hirabayashi S, Ogawa Y, Aota Y: Pedicle screws and bone mineral density. Spine 19:1157-1161, 1994

12. Kwon YM, Chin DK, Jin BH, Kim KS, Cho YE, Kuh SU: Long Term Efficacy of Posterior Lumbar Interbody Fusion with Standard Cages alone in Lumbar Disc Diseases Combined with Modic Changes. Journal of Korean Neurosurgical Society 46: 322-327, 2009

13. Lehman RA, Jr., Kuklo TR, Freedman BA, Cowart JR, Mense MG, Riew KD: The effect of alendronate sodium on spinal fusion: a rabbit model. The Spine Journal: Official Journal of the North American Spine Society 4:36-43, 2004

14. Lund T, Oxland TR, Jost B, Cripton P, Grassmann S, Etter $\mathrm{C}$, et al: Interbody cage stabilisation in the lumbar spine: biomechanical evaluation of cage design, posterior instrumentation and bone density. The Journal of Bone and Joint Surgery British Volume 80:351-359, 1998

15. McAfee PC, Boden SD, Brantigan JW, Fraser RD, Kuslich SD, Oxland TR, et al: Symposium: a critical discrepancy-a criteria of successful arthrodesis following interbody spinal fusions. Spine 26:320-334, 2001

16. Modic MT, Masaryk TJ, Ross JS, Carter JR: Imaging of degenerative disk disease. Radiology 168:177-186, 1988

17. Modic MT, Steinberg PM, Ross JS, Masaryk TJ, Carter JR: Degenerative disk disease: assessment of changes in vertebral body marrow with MR imaging. Radiology 166:193-199, 1988

18. Nagahama K, Kanayama M, Togawa D, Hashimoto T, Minami A: Does alendronate disturb the healing process of posterior lumbar interbody fusion? A prospective randomized trial. Journal of Neurosurgery Spine 14:500-507, 2011

19. Nakao S, Minamide A, Kawakami M, Boden SD, Yoshida M: The influence of alendronate on spine fusion in an osteoporotic animal model. Spine 36:1446-1452, 2011

20. Parfitt AM: The mechanism of coupling: a role for the vascula- 
ture. Bone 26:319-323, 2000

21. Sama AA, Khan SN, Myers ER, Huang RC, Cammisa FP, Jr., Sandhu HS, et al: High-dose alendronate uncouples osteoclast and osteoblast function: a study in a rat spine pseudarthrosis model. Clinical Orthopaedics and Related Research:135-142, 2004

22. Santos ER, Goss DG, Morcom RK, Fraser RD: Radiologic assessment of interbody fusion using carbon fiber cages. Spine 28: 997-1001, 2003

23. Sato M, Grasser W, Endo N, Akins R, Simmons H, Thompson $\mathrm{DD}$, et al: Bisphosphonate action. Alendronate localization in rat bone and effects on osteoclast ultrastructure. The Journal of Clinical Investigation 88:2095-2105, 1991

24. Selby P: Alendronate treatment for osteoporosis: a review of the clinical evidence. Osteoporosis international: a journal established as result of cooperation between the European Foundation for Osteoporosis and the National Osteoporosis Foundation of the USA 6:419-426, 1996

25. Siambanes D, Mather S: Comparison of plain radiographs and CT scans in instrumented posterior lumbar interbody fusion.
Orthopedics 21:165-167, 1998

26. Takahata M, Ito M, Abe Y, Abumi K, Minami A: The effect of anti-resorptive therapies on bone graft healing in an ovariectomized rat spinal arthrodesis model. Bone 43:1057-1066, 2008

27. Xue Q, Li H, Zou X, Bunger M, Egund N, Lind M, et al: Healing properties of allograft from alendronate-treated animal in lumbar spine interbody cage fusion. European spine journal : official publication of the European Spine Society, the European Spinal Deformity Society, and the European Section of the Cervical Spine Research Society 14:222-226, 2005

28. Xue Q, Li H, Zou X, Bunger M, Egund N, Lind M, et al: The influence of alendronate treatment and bone graft volume on posterior lateral spine fusion in a porcine model. Spine 30: 1116-1121, 2005

29. Xue QY, Ji Q, Li HS, Zou XN, Egund N, Lind M, et al: Alendronate treatment does not inhibit bone formation within biphasic calcium phosphate ceramics in posterolateral spinal fusion: an experimental study in porcine model. Chinese Medical Journal 122:2770-2774, 2009 